\title{
ERK activation in noradrenergic and serotonergic brainstem nuclei in chronic pain upon noxious stimulation and antidepressants treatment
}

\author{
G. Borges*, E. Berrocoso**, A. Ortega-Alvaro**,J. A. Micó**, F. L. Neto*
}

* Instituto de Histologia e Embriologia, Faculdade de Medicina do Porto e Instituto de Biologia Molecular e Celular (IBMC), Universidade do Porto, Portugal

** Departamento de Neurociencias (Farmacología y Psiquiatria), Grupo de Investigación y

Dessarollo en Neuropsicofarmacología, Facultad de Medicina, Universitad de Cádiz, Cádiz, España giselaborges90@gmail.com

Chronic neuropathic pain is a pathology that affects thousands of people worldwide. Antidepressants have been prescribed for the treatment of this sort of pain but the mechanisms underlying their analgesic action remain unknown [1]. Extracellular-signal regulated kinases (ERKs) are being implicated in pain transmission and modulation as well as in the pathophysiology of depression [2, 3]. In order to clarify some of the mechanisms which might be related to the analgesic effect of antidepressants, we started by evaluating possible changes in the pattern of activation of ERKs in rats with chronic constriction injury (CCI), an experimental model of chronic neuropathic pain [4], subjected either to noxious stimulation or to antidepressants treatment.

CCI was surgically induced by loose ligation of the sciatic nerve [4]. Control rats were only shamoperated. To evaluate the effect of noxious stimulation on ERKs activation, sham (sham-stimulated; SHAM-S) and CCI (CCI-stimulated; CCI-S) groups of anesthetised rats received a mechanical noxious stimulus within the area of the hind paw innervated by the sciatic nerve. Other two groups of sham and CCI rats did not receive any stimuli (SHAM-NS and CCI-NS, respectively). To study the influence of two antidepressants, CCI or SHAM rats were each divided in three groups and saline, amytriptyline (AMI, a tricyclic antidepressant) or duloxetine (DLX, a serotonin and norepinephrine reuptake inhibitor) were administered orally for 21 days. The daily amount of antidepressant diluted in water was evaluated for each rat. Hyperalgesia was analysed by the plantar and the paw pinch behavioural nociceptive tests. Immunoreactivity against pERKs1/2 was performed in $40 \mu \mathrm{m}$ paraformaldehyde perfusion-fixed brain slices [2] and immunoreactive cells (Fig. 1) were counted in monoaminergic brainstem nuclei.

Both SHAM-NS and SHAM-S rats showed baseline labelling for pERKS in some brainstem areas implicated in descending pain control mechanisms, like the A5 group of cells, Locus Coeruleus (LC), Rostroventromedial Medulla (including Lateral Paragintocellular - LPGi), Ventrolateral Medulla (including Lateral Reticular - LRt) and Dorsal Raphe (DR). In CCI rats, either stimulated or not- stimulated, the number of pERKs immunoreactive cells was increased in the A5, LPGi and LRt while in the LC the labelling intensity for pERKs decreased (Fig. 2), but in A5 and LC, changes between individual experimental groups did not reach statistical significance on post-hoc Bonferroni tests. No changes were detected between stimulated and non-stimulated rats, either SHAM or CCI. Antidepressants administration in SHAM animals induced significant changes in pERKs expression in some brainstem monoaminergic regions (Fig. 3). Thus, in A5, pERKs expression was increased in SHAM-AMI animals when compared with SHAM-saline group, while in LPGI either administration of AMI or DLX in SHAM rats significantly increased pERKs expression in comparison to saline treatment. Among CCI rats, administration of antidepressants had no effect on ERKs activation in any region studied. However, significant decreases were detected in CCI-amitriptyline when 
compared to SHAM-amitriptyline rats, in A5 and in DR. No differences were detected for DLX administration.

Data suggests noxious stimulation has no significant effect on the expression of pERKs1/2 while the occurrence of a neuropathic painful condition changed the pattern of ERKs activation in some brainstem regions. Moreover, under normal conditions antidepressants seem to act on ERKs pathway, most probably in nuclei with serotonergic and/or noradrenergic characteristics, but under chronic painful input these mechanisms seem altered. More studies are needed in order to clarify the circuit where antidepressants act to induce analgesia and the role of ERKs.

\section{References:}

[1] J. A. Micó, D. Ardid, E. Berrocoso, A. Eschalier A. Trends Pharmacol Sci, 27(7) (2006) 348.

[2] C. D. Cruz, F.L. Neto, J. Castro-Lopes, S B McMahon, F. Cruz, Pain, 116(3) (2005) 411.

[3] F. Fumagalli, R. Molteni, F Calabrese, A. Frasca, G. Racagni, M. A. Riva. J Neurochem, 93(6) (2005) 1551.

[4] G. J. Bennett, Y. K. Xie. Pain, 33(1) (1988) 87.

Granted by PI070687 and HP2006-0062, Spain and Acção Integrada E-42/07 from CRUP, Portugal
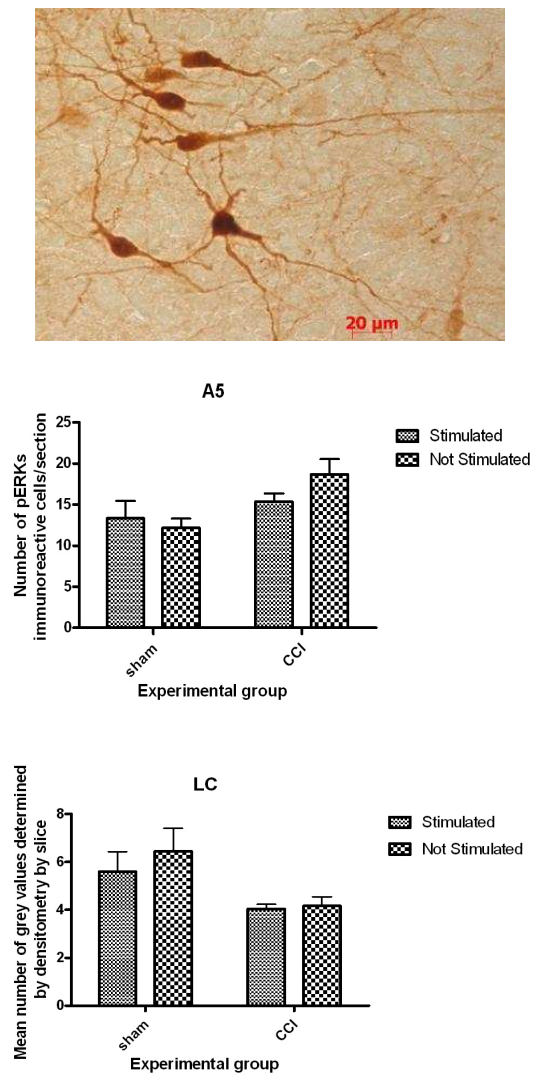

Fig 3 - Effect of neuropathic pain and antidepressants treatment on the number of cells expressing pERKs in A5 and LPGi. Number of pERK IRcells expressed as mean \pm S.E.M. \# and * represent post-hoc Bonferroni statistic differences for factors "surgery" and "drug", respectively, in two-way ANOVA. \#p <0.05; * p $<0.05 ; * * \mathrm{p}<0.01$.

Fig 1 - Photomicrographs showing pERKs1/2 labelling. Immunoreactivity is mainly located in cytoplasm and, sometimes, in nuclei.
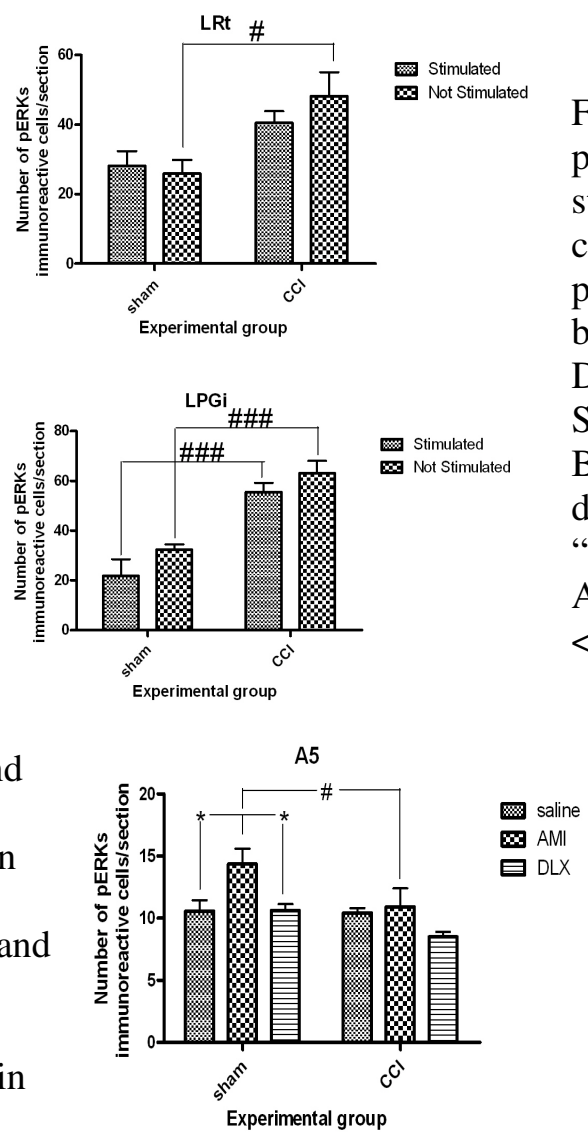

Fig 2 - Effect of neuropathic pain and noxious mechanical stimulation on the number of cells or labeling intensity of pERKs expression in main brainstem areas examined. Data expressed by mean \pm S.E.M. \# represents post-hoc Bonferroni statistic differences for factor "surgery" in two-way ANOVA. \#p $<0.05 ; \#$ p $<0.01 ; \# \#$ \# $<0.001$.

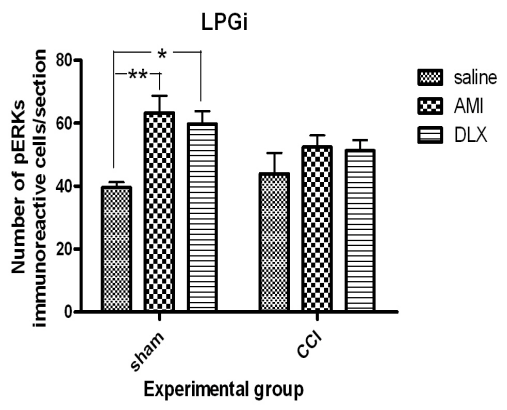

